\title{
Perioperative anaphylaxis and investigations: a local study in Hong Kong
}

\author{
Elaine Yuen Ling $\underline{A u^{1}}$, MBBS, FHKCPath, Chak Sing $\underline{L a u^{2}}$, MBChB, MD(Hons), Ki $\underline{L a m}^{1}$, MBBS, Eric $\underline{C h a n^{1}}$, MBBS, PhD
}

INTRODUCTION Data on local intraoperative anaphylaxis in Hong Kong is scarce, with few reviews available. We aimed to study the characteristics, presentations and workup results of cases referred to a local allergy clinic.

METHODS A retrospective review was performed of patient referrals and workup results for suspected intraoperative anaphylaxis at Queen Mary Hospital drug allergy clinic in 2012-2016.

RESULTS Tryptase was checked in only $81.7 \%$ (49/60) of the cases, most of which showed elevation (71.4\%, 35/49). Among the 59 patients who received a workup, 47 (79.7\%) showed positive findings, with a particularly high yield in the tryptase-positive subgroup $(88.6 \%, 31 / 35)$. Among the 54 patients who consented to skin tests (the most sensitive investigation), 43 (79.6\%) cases were positive. Overall, neuromuscular blockers were the commonest cause (25.0\%, 15/60) of intraoperative anaphylaxis, while antibiotics ranked second $(23.3 \%, 14 / 60)$. The timing of reactions was an important indication of potential allergens. For example, the majority of the neuromuscular blocker allergies occurred during the induction phase, while all gelofusine allergic events were in the maintenance phase of anaesthesia. 13 (21.7\%) out of 60 cases received subsequent general anaesthesia procedures, with no recurrence of allergic reactions.

CONCLUSION Proper workup after an intraoperative anaphylactic event has a fairly good chance of identifying the causative allergen. These results are useful for patient management and the planning of subsequent anaesthetic procedures.

Keywords: basophil activation test, Hong Kong, intraoperative anaphylaxis, skin test, specific lgE

\section{INTRODUCTION}

During anaesthesia, patients are exposed to a number of drugs and substances, and unforeseen severe reactions may occur. Anaphylaxis is a severe, life-threatening, generalised, systemic, acute allergic reaction, usually triggered by immunoglobulin $\mathrm{E}$ (IgE)-dependent release of mediators from mast cells or basophils. The incidence of perioperative anaphylaxis was reported to be one out of 10,000-20,000 operations. ${ }^{(1-4)}$ Nevertheless, the true incidence of anaphylactic reactions and their associated morbidity and mortality remains poorly defined, as there are variations in diagnostic accuracy and reporting methodology.

Recognition of an allergic event during anaesthesia may not be straightforward. Anaphylaxis is not a homogeneous process, and patients' presentations vary according to the triggering agent, dose and route of exposure, and underlying comorbid conditions or other concomitant drug use. In addition, patients are sedated and under drapes. Early cutaneous signs may go undetected, although the absence of cutaneous signs does not exclude the diagnosis. Usually, the event is recognised when the patient presents with systemic upset with bronchospasm or cardiovascular collapse. One must also be aware that other complications, (e.g. cardiovascular events and thromboembolism) may mimic anaphylaxis, with a similar presentation during anaesthesia.

Common causative agents of perioperative anaphylaxis include neuromuscular blockers, latex and antibiotics. ${ }^{(1-4)}$ The diagnosis of perioperative anaphylaxis relies on history and clinical findings, and is supported by documentation of elevated tryptase levels during the event. Serum tryptase is a marker for mast cell degranulation, although it does not differentiate between $\operatorname{IgE}$ and non-IgE mediated events. Moreover, negative tryptase results do not exclude anaphylaxis.

The workup of perioperative anaphylaxis is often complicated. The multiple drugs that are administered during anaesthesia may elicit various allergic or non-allergic reactions. In addition, nonanaesthesia-related drugs or procedures during the operation (e.g. disinfectants and latex) may also be the causative agent of the allergic reactions. None of the available diagnostic tests are perfect. False positive or false negative results do exist. There is no 'gold standard' to verify the findings unless the patient is exposed to the agents again, which is risky and may not be feasible. Conventionally, a skin test using the prick or intradermal techniques is used in the workup. In vitro tests such as the specific $\lg \mathrm{E}(\mathrm{S} \lg \mathrm{E})$ test and basophil activation test (BAT) may also be considered.

In Hong Kong, there is a lack of reviews of the clinical laboratory profile of allergic reactions during anaesthesia. Hence, we performed a retrospective review of tertiary allergy clinic referrals for intraoperative allergy from 2012-2016.

\section{METHODS}

We conducted a retrospective chart review of referrals to a tertiary allergy clinic. The study was approved by the hospital's institutional review board. All patients referred to the drug allergy clinic of Queen Mary Hospital in 2012-2016 for recent intraoperative anaphylaxis and aged $\geq 18$ years were included

${ }^{1}$ Division of Clinical Immunology, Department of Pathology, Queen Mary Hospital, ${ }^{2}$ Department of Medicine, Queen Mary Hospital, Li Ka Shing Faculty of Medicine, the University of Hong Kong, Hong Kong

Correspondence: Dr Elaine Au Yuen Ling, Consultant, Division of Clinical Immunology, Department of Pathology, Room 4, 14/F, Block T, Queen Mary Hospital, 102 Pokfulam Road, Hong Kong. elaineauyl@gmail.com 
in the study. Queen Mary Hospital drug allergy clinic provides a territory-wide drug allergy workup service for adult patients. The clinic receives referrals from other public and private hospitals. The diagnostic workup includes history and clinical assessment, blood tests (SIgE test and BAT) and skin tests. Patients referred for recent perioperative anaphylaxis were given early assessment, with skin tests and blood tests done within six weeks to six months.

Patients who had suspected anaphylaxis during the perioperative period in the operating theatre or recovery room were included in the analysis. The clinical diagnosis for referral to the allergy clinic was made by the attending anaesthetists, who based their judgement on clinical grounds and exclusion of disease entities mimicking intraoperative anaphylaxis. The medical records of these patients were retrieved and studied. Demographic data on age, gender, previous anaesthesia and anaesthesia exposure before the index event, clinical presentation, and timing of the reactions were recorded. Cardiovascular complications were also recorded, with hypotension being defined as systolic blood pressure $<90 \mathrm{mmHg}$. Bronchospasm was defined as clinical evidence of airway obstruction and wheezing diagnosed by the attending anaesthetist. Skin flushing and rash were seen as allergic manifestations according to the clinical judgement of the attending anaesthetist. Tryptase results and the allergy workup findings, including results from skin tests, SIgE test and BAT, were reviewed. The outcomes of subsequent operations and anaesthesia after the index event were also studied.

For those patients whose tryptase levels were taken, two blood samples were sent to the laboratory. The acute phase sample was taken within 30-120 minutes of the event, while the second sample was taken at least 24 hours post event to serve as the baseline. Levels of serum tryptase were measured using fluoroimmunoassay (ImmunoCAP Tryptase, Phadia Laboratory Systems, Sweden). A reaction level of at least $2 \mathrm{ng} / \mathrm{mL}+(1.2 \times$ post-reaction tryptase level $)$ was defined as elevated tryptase level. ${ }^{(5)}$

All patients were offered skin tests, including prick and intradermal testing on a battery of drugs and substances that they had been exposed to during the operation before the event. Histamine $10 \mathrm{mg} / \mathrm{mL}$ and saline solution were used as positive and negative controls, respectively. Skin tests started with the prick test and proceeded to intradermal tests using increasing concentrations of drugs, up to non-irritating concentrations reported in the literature. ${ }^{(6-11)}$ Readings were taken after 20 minutes and assessed according to the drug allergy practice parameter by the American Academy of Allergy, Asthma and Immunology. ${ }^{12}$ Skin tests were regarded as positive or negative according to the interpretation parameters.

BAT was done using flow cytometry: blood samples were incubated with suspected allergens, and activation markers of basophil (i.e. CD63/CD203) were subsequently measured. CD63 is normally expressed inside the vesicle membranes where histamine is stored. IgE-mediated degranulation leads to the expression of this marker on the external surface of basophils. Stimulation buffer containing interleukin-3 was used to enhance CD63 expression. A Buhlmann kit (Buhlmann Diagnostic,
Sweden) and drugs from the pharmacy were employed. The SIgE test was done using fluoroimmunoassay (ImmunoCap Tryptase, Phadia Laboratory Systems, Sweden). SIgE against amoxicilloyl, ampicilloyl, penicilloyl G, penicilloyl V, pholcodine, suxamethonium, chlorhexidine, gelatin and morphine were available in the immunology laboratory. These tests had been arranged as workup according to the case and exposure history. The attending immunologist in the allergy clinic assessed patients' clinical history and allergy workup holistically to identify a composite allergy diagnosis and the causative agent.

For cases with anaphylaxis during the induction, maintenance and recovery phases, descriptive analysis was performed of demographic, clinical and laboratory parameters. Comparisons were performed using non-parametric Mann-Whitney $U$ test and Fisher's exact test. A p-value $<0.05$ was considered to be statistically significant. Characteristics between tryptase-positive and -negative groups were contrasted and compared. IBM SPSS Statistics for Windows version 22.0 (IBM Corp, Armonk, NY, USA) was utilised for statistical analysis.

\section{RESULTS}

During the study period, 60 patients (33 female, 27 male) with perioperative hypersensitivity reactions were referred for workup during the postoperative follow-up period. Their mean age was 55.9 (range 19-88) years. More than half (55.0\%) of the patients in this cohort suffered from suspected intraoperative anaphylaxis during their first anaesthesia, with ten cases developing the reaction during their first anaesthetic exposure. Most (58.3\%, $\mathrm{n}=35$ ) reactions happened during the induction period, with $14(23.3 \%)$ reactions during maintenance and $6(10.0 \%)$ reactions in the recovery phase (Table I). Five non-general anaesthesia cases, the majority being spinal anaesthesia cases, were referred for suspected anaphylaxis workup (Table II).

Out of the referred cases, $87.3 \%$ suffered from severe reactions, presenting with hypotension, while $45.5 \%$ presented with bronchospasm and wheeze. On the contrary, rash, a common symptom of allergy, was noted in less than half (47.3\%) of all cases. There were more cases with hypotension and shock due to anaphylaxis during the induction and maintenance periods (Table I \& Fig. 1). Tryptase was not checked in 11 (18.3\%) cases. Out of the 49 cases who had tryptase checked, most $(71.4 \%$, $\mathrm{n}=35$ ) showed elevation. After referral, one patient declined further investigation. Among the 59 patients who underwent skin and blood tests, $79.7 \%(n=47)$ demonstrated positive findings, while the other results were unrevealing. Elevated tryptase correlated with a positive workup. Up to $88.6 \%$ of tryptase-elevated cases eventually had a positive workup, significantly higher than the $42.9 \%$ among the tryptase-negative cases (Table III). Among the 11 cases who did not have tryptase checked, $90.9 \%(n=10)$ yielded positive findings. Skin test was the most sensitive investigation. Among the 54 patients who consented to skin tests, 43 (79.6\%) cases were positive. For the in vitro test, BAT had higher sensitivity than SIgE. The detection rate of BAT was $31.6 \%$ (18/57), while SIgE only detected $18.5 \%$ $(10 / 54)$ of cases. Overall, the sensitivity of SIgE with reference to 
Table I. Clinical characteristics and allergy workup of patients with intraoperative anaphylaxis during different phases of general anaesthesia.

\begin{tabular}{|c|c|c|c|c|c|}
\hline \multirow[t]{2}{*}{ Parameter } & \multicolumn{4}{|c|}{ No. (\%) } & \multirow[t]{2}{*}{ p-value } \\
\hline & All $(n=55)$ & $\begin{array}{l}\text { Induction phase } \\
(\mathrm{n}=35)\end{array}$ & $\begin{array}{l}\text { Maintenance phase } \\
(n=14)\end{array}$ & $\begin{array}{l}\text { Recovery phase } \\
(n=6)\end{array}$ & \\
\hline Age* $(y r)$ & $60(19-88)$ & $60(23-76)$ & $62(19-88)$ & $57(19-79)$ & 0.732 \\
\hline Male gender & $25(45.5)$ & $14(40.0)$ & $6(42.9)$ & $5(83.3)$ & 0.163 \\
\hline Previous anaesthesia & $24(43.6)$ & $11(31.4)$ & $8(57.1)$ & $5(83.3)$ & 0.030 \\
\hline \multicolumn{6}{|l|}{ Clinical manifestation } \\
\hline Bronchospasm & $25(45.5)$ & $19(54.3)$ & $5(35.7)$ & $1(16.7)$ & 0.179 \\
\hline Hypotension $^{\dagger}$ & $48(87.3)$ & $33(94.3)$ & $13(92.9)$ & $2(33.3)$ & 0.002 \\
\hline Skin rash/flushing & $26(47.3)$ & $15(42.9)$ & $6(42.9)$ & $5(83.3)$ & 0.200 \\
\hline Tryptase elevation & $34 / 48(70.8)$ & $21 / 32(65.6)$ & $11 / 14(78.6)$ & $2 / 2(100.0)$ & 0.538 \\
\hline \multicolumn{6}{|l|}{ Allergy workup } \\
\hline Positive skin test & $39 / 51(76.5)$ & $25 / 33(75.8)$ & 9/12 (75.0) & $5 / 6(83.3)$ & 1.000 \\
\hline Positive SlgE test & $8 / 49(16.3)$ & $6 / 30(20.0)$ & $1 / 13(7.7)$ & $1 / 6(16.7)$ & 0.748 \\
\hline Positive BAT & $17 / 52(32.7)$ & $8 / 33(24.2)$ & $7 / 14(50.0)$ & $2 / 5(40.0)$ & 0.187 \\
\hline Composite allergy diagnosis & $43 / 55(78.2)$ & $26 / 35(74.3)$ & $11 / 14(78.6)$ & $6 / 6(100.0)$ & 0.546 \\
\hline Causative agent identified & $\mathrm{n}=52,43$ patients & $\mathrm{n}=33,26$ patients & $\mathrm{n}=13,11$ patients & $\mathrm{n}=6,6$ patients & - \\
\hline \multicolumn{6}{|l|}{ Neuromuscular blocker } \\
\hline Total (by agent) & $20 / 52(38.5)$ & $18 / 33(54.5)$ & $1 / 13(7.7)$ & $1 / 6(16.7)$ & \\
\hline Total (by patient) & $15 / 55(27.3)$ & $13 / 35(37.1)$ & $1 / 14(7.1)$ & $1 / 6(16.7)$ & \\
\hline Non-depolarising agent & $11 / 55(20)$ & 9/35 (25.7) & $1 / 14(7.1)$ & $1 / 6(16.7)$ & \\
\hline Depolarising agent & 9/55 (16.4) & 9/35 (25.7) & $0(0)$ & $0(0)$ & \\
\hline Antibiotics & $14 / 55(25.5)$ & 10/35 (28.6) & $3(21.4)$ & $1 / 6(16.7)$ & \\
\hline \multicolumn{6}{|l|}{ Other medication } \\
\hline Midazolam & $3 / 55(5.5)$ & $3 / 35(8.6)$ & $0(0)$ & $0(0)$ & \\
\hline Esomeprazole & $1 / 55(1.8)$ & $1 / 35(2.9)$ & $0(0)$ & $0(0)$ & \\
\hline Ondansetron & $3 / 55(5.5)$ & $0(0)$ & $1 / 14(7.1)$ & 2/6 (33.3) & \\
\hline Gelofusine & $7 / 55(12.7)$ & $0(0)$ & $6 / 14(42.9)$ & $1 / 6(16.7)$ & \\
\hline \multicolumn{6}{|l|}{ Other chemical } \\
\hline Chlorhexidine & $1 / 55(1.8)$ & $0(0)$ & $0(0)$ & $1 / 6(16.7)$ & \\
\hline Latex & $1 / 55(1.8)$ & $0(0)$ & $1 / 14(7.1)$ & $0(0)$ & \\
\hline Patent blue dye & $2 / 55(3.6)$ & $1 / 35(2.9)$ & $1 / 14(7.1)$ & $0(0)$ & \\
\hline Subsequent anaesthesia & $13 / 55(23.6)$ & 10/35 (28.6) & $3 / 14(21.4)$ & $0(0)$ & \\
\hline Allergic event & $0(0)$ & $0(0)$ & $0(0)$ & $0(0)$ & - \\
\hline
\end{tabular}

Value of $\mathrm{n}$ is listed in cases where some data was unavailable, and percentages were calculated based on available data. *Data presented as median (range). + Defined as systolic blood pressure $<90 \mathrm{mmHg}$. BAT: basophil activation test; SlgE: specific immunoglobulin E

Table II. Cases of anaphylaxis induced during non-general anaesthesia procedures.

\begin{tabular}{|c|c|c|c|c|c|c|c|}
\hline Gender, age (yr) & $\begin{array}{l}\text { Previous } \\
\text { surgery }\end{array}$ & Type of reaction & Tryptase & BAT & SlgE test & Skin test & $\begin{array}{l}\text { Subsequent } \\
\text { anaesthesia }\end{array}$ \\
\hline Female, 33 & 0 & Cutaneous & NT & Negative & Negative & Syntocinon & No \\
\hline Female, 70 & 3 & Cutaneous, hypotension & NT & Negative & Chlorhexidine & Chlorhexidine & No \\
\hline Female, 78 & 2 & Bronchospasm & NT & Gelofusine & Negative & NT & No \\
\hline Male, 69 & 0 & Cutaneous, bronchospasm & Positive & Negative & Negative & Gelofusine & No \\
\hline Male, 69 & 1 & Cutaneous, hypotension & NT & Negative & Chlorhexidine & Chlorhexidine & No \\
\hline
\end{tabular}

BAT: basophil activation test; NT: not tested; SlgE: specific immunoglobulin E

positive skin test cases was only $23.3 \%(10 / 43)$ and that of BAT was $41.9 \%(18 / 43)$.

In our cohort of 60 patients, neuromuscular blockers were the commonest cause of intraoperative anaphylaxis $(25.0 \%, \mathrm{n}=15)$, while antibiotics ranked second $(23.3 \%, \mathrm{n}=14)$. All the antibiotic allergy cases in this cohort were caused by beta lactams, most commonly cefazolin, which is a common choice of induction antibiotic locally. Anaphylaxis caused by neuromuscular blockers usually occurred during the induction phase. A plasma expander, gelofusine, also constituted a significant number of anaphylaxis 


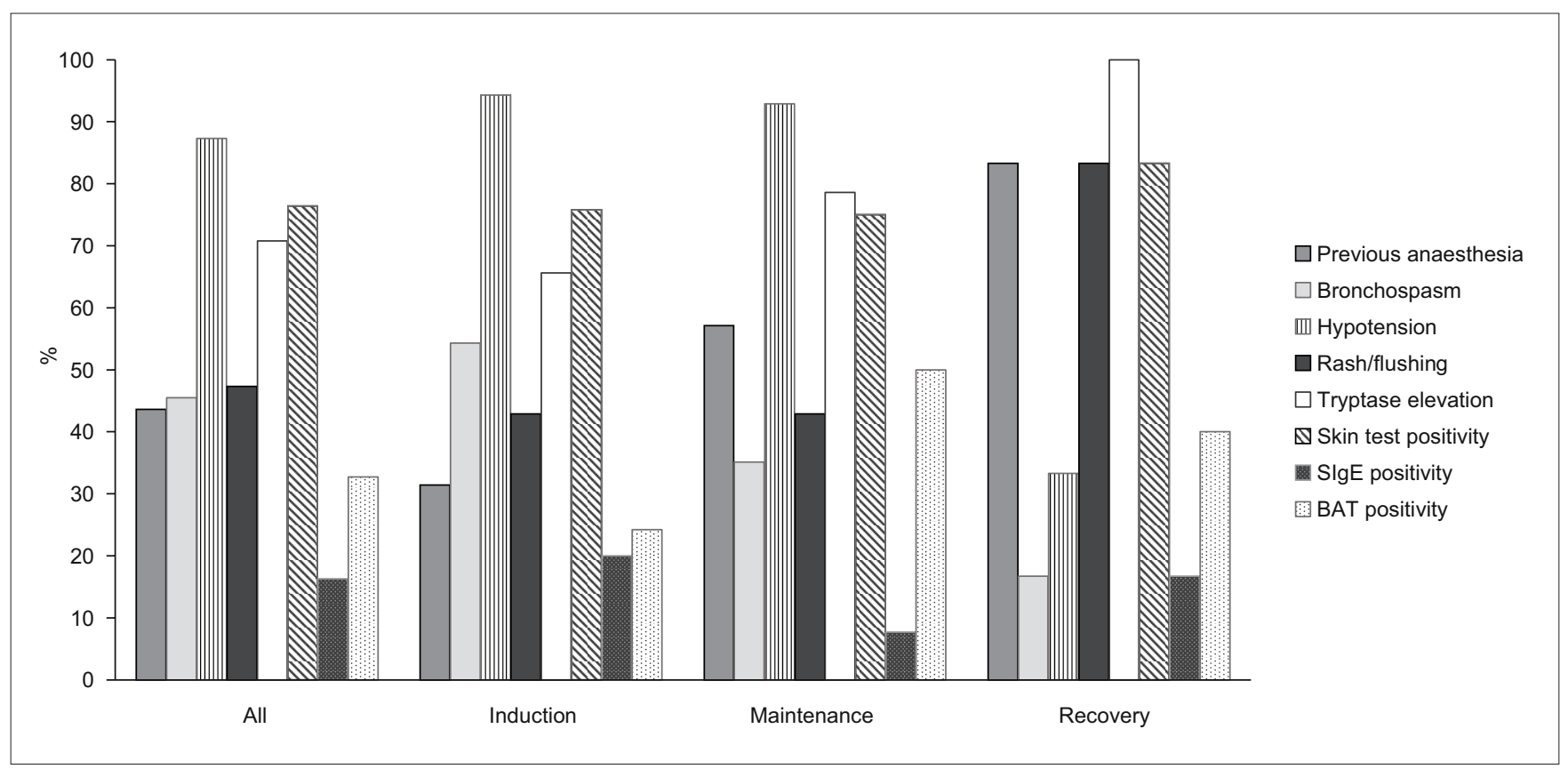

Fig. 1 Chart shows the clinical characteristics of patients with intraoperative anaphylaxis during general anaesthesia. BAT: basophil activation test; SIgE: specific immunoglobulin E

Table III. Comparison of the clinical characteristics of patients with tryptase-positive and -negative intraoperative anaphylaxis.

\begin{tabular}{|c|c|c|c|}
\hline \multirow[t]{2}{*}{ Characteristic } & \multicolumn{2}{|c|}{ No. (\%) } & \multirow[t]{2}{*}{ p-value } \\
\hline & Tryptase-positive $(n=35)$ & Tryptase-negative $(n=14)$ & \\
\hline Age* (yr) $^{*}$ & $60(26-88)$ & $62(19-76)$ & 0.859 \\
\hline Male gender & $13(37.1)$ & $8(57.1)$ & 0.201 \\
\hline Previous anaesthesia & $14(40.0)$ & $5(35.7)$ & 1.000 \\
\hline Time of onset in GA & & & 0.668 \\
\hline Maintenance & $11(31.4)$ & $3(21.4)$ & \\
\hline Recovery & $2(5.7)$ & $0(0)$ & \\
\hline Non GA & $1(2.9)$ & $0(0)$ & \\
\hline \multicolumn{4}{|l|}{ Clinical manifestation } \\
\hline Bronchospasm & $20(57.1)$ & $4(28.6)$ & 0.114 \\
\hline Skin rash/flushing & $16(45.7)$ & $6(42.9)$ & 0.856 \\
\hline \multicolumn{4}{|l|}{ Allergy workup } \\
\hline Positive skin test & $28(80.0)$ & $6(42.9)$ & 0.017 \\
\hline Positive SIgE test & $6(17.1)$ & $0(0)$ & 0.164 \\
\hline Positive BAT & $15(42.9)$ & $0(0)$ & 0.004 \\
\hline Composite allergy diagnosis & $31(88.6)$ & $6(42.9)$ & 0.002 \\
\hline
\end{tabular}

*Data presented as median (range). BAT: basophil activation test; GA: general anaesthesia; SlgE: specific immunoglobulin E

episodes $(15.0 \%, 9 / 60)$ in our cohort, which was the commonest cause of anaphylaxis during the maintenance phase in our study (Tables I \& II).

A total of $13(21.7 \%)$ out of 60 cases received subsequent general anaesthesia procedures, after which recurrence of allergic events was not observed. These patients suffered from severe reactions during the index events, with elevated tryptase in the majority $(69.2 \%, \mathrm{n}=9)$. Another $3(23.1 \%)$ patients had negative tryptase, and $1(7.7 \%)$ patient did not have tryptase checked. Most often $(76.9 \%, n=10)$, the anaesthetists avoided the allergen group in subsequent operations. Three cases were assessed to be allergic to neuromuscular blockers (i.e. suxamethonium; suxamethonium; and atracurium), and alternative neuromuscular blockers that were skin-test negative were given to the patients uneventfully (atracurium; rocuronium; and suxamethonium and rocuronium, respectively).

\section{DISCUSSION}

To our knowledge, this is the first study on intraoperative anaphylaxis cases in Hong Kong. Local data on intraoperative 
anaphylactic events is lacking, and the local incidence is currently unknown. It was interesting to note that more than half of the patients in our cohort suffered from suspected intraoperative anaphylaxis during their first anaesthesia (55.0\%). For example, among the 15 cases of neuromuscular blocker allergy, ten cases developed the reaction during their first anesthetic exposure. The finding that previous exposure history was found in less than half of the patients who were allergic to neuromuscular blockers is consistent with the literature. ${ }^{(13)}$ On the other hand, a past history of uneventful exposure may sensitise an individual and result in reactions upon re-exposure.

Differentiating anaphylaxis from other mimics in intraoperative settings is no easy task. Although retrospective reviews of medical records may result in possible inaccuracies in terms of symptom assessment, the clinical manifestations observed in this cohort were in accordance with those reported in the literature, indicating that cutaneous manifestations are not as common or obvious as other allergic reactions, such as those reported in non-intraoperative settings. The fact that patients in our cohort were sedated and under drapes may explain the observation that cutaneous manifestations were more frequently noticed during the recovery phase than the induction/maintenance phase of general anaesthesia. Unexplained refractory shock or clinical evidence of acute bronchospasm is frequently encountered without prior warning signals during general anaesthesia. Therefore, anaphylaxis should always be considered as a possible differential diagnosis in these situations, and appropriate management should be started without delay. Although tryptase testing does not affect immediate management, the results do facilitate the subsequent workup and provide useful information for future anaesthesia planning.

The workup of perioperative anaphylaxis is often complicated. The multiple drugs administered during anaesthesia may elicit various allergic or non-allergic reactions. In addition, nonanaesthesia-related drugs or procedures during the operation (e.g. disinfection and latex) can be the cause of the allergic reaction. Identifying the culprit allergen is important for subsequent operation and anaesthetic procedures. Previous studies have demonstrated the usefulness of skin tests in the workup. $^{(14,15)}$ In general, skin tests are more sensitive than blood tests but also have their limitations. Firstly, they re-expose patients to suspected allergens, which entails a small potential risk to the patients. Moreover, interpretation of skin tests could be difficult in some skin conditions and in patients taking certain medications such as antihistamines and tricyclic antidepressants. Hence, there is a role for blood tests to complement skin tests in the workup, on top of careful history-taking and review of previous anaesthetic records. In our study, assessments were made using skin and blood tests, including SIgE and BAT. Among the patients who had been referred to us and had agreed to further investigations, $79.7 \%$ had positive workup results. Hence, a comprehensive assessment integrating clinical history, skin tests and blood tests provides a fairly good chance of identifying the suspected allergens, which would be important for future anaesthesia planning and patient care.
Among these investigations, the skin test was the most sensitive workup. Overall, the sensitivity of SIgE with reference to a positive skin test was only $23.3 \%$, and that of BAT was about $41.9 \%$. Among the five patients who declined a skin test assessment, three had a gelofusine allergy identified by BAT. Additionally, up to $88.6 \%$ of tryptase-elevated cases finally resulted in a positive workup, compared to $42.9 \%$ of the tryptase-negative cases. It is likely that the tryptase-elevated cases were more severe, providing a definite diagnosis of anaphylaxis. Indeed, the majority of the referred cases presented with haemodynamic instability and a clinical diagnosis of anaphylaxis. Nevertheless, it is possible that other non-allergy-related medical conditions contributed to the clinical presentation in the tryptase-negative and workupnegative cases. Notably, BAT failed to yield positive results in all tryptase-negative cases but showed positive results in up to $42.9 \%$ of tryptase-elevated cases. Hence, our findings suggest that the utility of BAT in documented tryptase-negative cases is probably minimal. Nevertheless, prospective studies with a larger sample size are needed for more definite conclusions on the use of BAT in such cases. Overall, the yield of the SIgE test was low, and one should not base patient management decisions solely on the result of this investigation.

In our cohort, neuromuscular blockers were the most frequent aetiological agents identified, followed by beta-lactam antibiotics, which is similar to previous findings that neuromuscular blockers and antibiotics are the two commonest causative agents in intraoperative anaphylaxis. ${ }^{(16-18)}$ However, in our cohort, latex allergy was not as prevalent as in previous studies. ${ }^{(16-18)}$ Another interesting finding was that gelofusine, a commonly used plasma expander, was the commonest cause of anaphylaxis in the maintenance phase. Being alert to the possibility of gelofusine allergy is important, since gelofusine is commonly employed in the management of haemodynamic instability, a possible manifestation of anaphylaxis. It is also important to note that non-drug items such as patent blue dye and chlorhexidine may induce anaphylaxis, hence we should be vigilant to such potential allergens, especially in workup cases that appear negative. As noted in our study, the timing of the allergic reaction provides a clue to the potential allergen. For example, most neuromuscular blocker allergies occurred in the induction phase, while all gelofusine allergies took place during the maintenance/recovery phase of general anaesthesia.

Although only a small percentage of cases in our study underwent subsequent anaesthetic procedures, recurrence of allergic events was not observed, as the suspected allergen groups identified in the workup were avoided. Three cases with a past history of reaction towards neuromuscular blockers had uneventful general anaesthesia using alternative, skin-test negative neuromuscular blockers. This supports findings in the literature that skin-test-negative neuromuscular blocker alternatives may be considered in patients with past allergic history. ${ }^{(19,20)}$

Our study was limited by its retrospective nature. Furthermore, not all patients completed all the investigations. Moreover, due to limitations in resources and feasibility, we did not perform drug challenge tests to verify negative workup items. Nevertheless, to 
our knowledge, our study is the first to provide an overview of intraoperative anaphylaxis in Hong Kong using both skin tests and blood test assessments.

In conclusion, a comprehensive allergy assessment integrating clinical history, skin tests and blood tests has a good chance of identifying possible causative allergens, which is important for future anaesthesia planning and patient management.

\section{REFERENCES}

1. Fisher MM, Baldo BA. The incidence and clinical features of anaphylactic reactions during anesthesia in Australia. Ann Fr Anesth Reanim 1993; 12:97-104

2. Watkins J. Adverse reaction to neuromuscular blockers: frequency, investigation, and epidemiology. Acta Anaesthesiol Scand Suppl 1994; 102:6-10.

3. Vervloet D, Magnan A, Birnbaum J, Pradal M. Allergic emergencies seen in surgical suites. Clin Rev Allergy Immunol 1999; 17:459-67.

4. Fasting S, Gisvold SE. [Serious intraoperative problems--a five-year review of 83,844 anesthetics]. Can J Anaesth 2002; 49:545-53.

5. De Schryver S, Halbrich M, Clarke A, et al. Tryptase levels in children presenting with anaphylaxis: temporal trends and associated factors. J Allergy Clin Immunol 2016; 137:1138-42.

6. Brockow K, Garvey LH, Aberer W, et al; ENDA/EAACI Drug Allergy Interest Group. Skin test concentrations for systemically administrated drug--an ENDA EAACI Drug Allergy Interest Group position paper. Allergy 2013; 68:702-12.

7. Michavila Gomez AV, Belver Gonzalez MT, Alvarez NC, et al; Drug allergy Work Group of the Spanish Society of Paediatric Allergy, Immunology (SEICAP). Perioperative anaphylactic reactions: review and procedure protocol in paediatrics. Allergol Immunopathol (Madr) 2015; 43:203-14.

8. Ebo DG, Fisher MM, Hagendorens MM, Bridts CH, Stevens WJ. Anaphylaxis during anaesthesia: diagnostic approach. Allergy 2007; 62:471-87.

9. Romano A, Caubet JC. Antibiotic allergies in children and adults: from clinical symptoms to skin testing diagnosis. J Allergy Clin Immunol Pract 2014; 2:3-12.

10. Apostolou E, Deckert K, Puy R, et al. Anaphylaxis to gelufusine confirmed by in vitro basophil activation test: a case series. Anaesthesia 2006; 61:264-8.

11. Kepil Özdemir S, Yılmaz I, Aydin Ö, et al. Immediate-type hypersensitivity reactions to proton pump inhibitors: usefulness of skin tests in the diagnosis and assessment of cross-reactivity. Allergy 2013; 68:1008-14.

12. Joint Task Force on Practice Parameters; American Academy of Allergy, Asthma and Immunology; American College of Allergy, Asthma and Immunology; Joint Council of Allergy, Asthma and Immunology. Drug allergy: an updated practice parameter. Ann Allergy Asthma Immunol 2010; 105:259-73.

13. Harper NJ, Dixon T, Dugué $P$, et al; Working Party of the Association of Anaesthetists of Great Britain and Ireland. Suspected anaphylactic reactions associated with anaesthesia. Anaesthesia 2009; 64:199-211.

14. Baldo BA, Pham NH, Zhao Z. Chemistry of drug allergenicity. Curr Opin Allergy Clin Immunol 2001; 1:327-35

15. Brockow K, Romano A, Blanca M, et al. General considerations for skin test procedures in the diagnosis of drug hypersensitivity. Allergy 2002; 57:45-51.

16. Harboe T, Guttormsen AB, Irgens A, Dybendal T, Florvaag E. Anaphylaxis during anesthesia in Norway: a 6-year single-center follow-up study. Anesthesiology 2005; 102:897-903.

17. Lieberman P. Anaphylactic reactions during surgical and medical procedures. J Allergy Clin Immunol 2002; 110(2 Suppl):S64-9.

18. Hepner DL, Castells MC. Anaphylaxis during the perioperative period. Anesth Analg 2003; 97:1381-95.

19. Villas Martínez F, Joral A, Garmendia FJ, Navarro JA. Anaphylactic reactions to suxamethonium (succinylcholine). J Investig Allergol Clin Immunol 1999; 9:126-8.

20. Dewachter P, Mouton-Faivre C, Pertek JP, Boudaa C, Mertes PM. [Value of skin tests for the choice of a neuromuscular blocking agent after an anaphylactic reaction]. Ann Fr Anesth Reanim 2005; 24:543-6. French. 